\title{
Mapping quantitative trait loci controlling low-temperature germinability in rice
}

\author{
Nguyen Hoang Nam ${ }^{1}$, In-Kyu Park ${ }^{1}$, Sang-Min Yeo ${ }^{1}$, Yeo-Tae Yun ${ }^{2}$, Sang-Nag Ahn ${ }^{1 *}$ \\ ${ }^{1}$ Department of Agronomy, College of Agriculture and Life Sciences, Chungnam National University, Daejeon 305-764, Korea \\ ${ }^{2}$ Chungnam Agricultural Research and Extension Services, Yesan 340-861, Korea
}

Received on 23 October 2012, revised on 10 December 2012, accepted on 10 December 2012

\begin{abstract}
Low-temperature germination is one of the major determinants for stable stand establishment in the rice direct seeding method in temperate regions and at high altitude areas. Quantitative trait loci (QTL) controlling low-temperature germinability in rice were identified using 96 introgression lines (ILs) derived from a cross between Oryza rufipogon and the Korean japonica cultivar, 'Hwaseongbyeo'. The germination rate at $15^{\circ} \mathrm{C}$ was measured to represent low-temperature germination and used for QTL analysis. The germination rate at $15^{\circ} \mathrm{C}$ for 7 days of Oryza rufipogon and Hwaseongbyeo was 93.3 and $28.7 \%$, respectively, and that of progenies ranged from 0 to $48 \%$. A linkage map was constructed using 135 simple sequence repeat (SSR) markers. Five putative QTLs associated with low-temperature germination were detected on chromosomes 1, 3, 4, 10 and 11. The QTL, qltg10 on chromosome 10 accounted for $19.2 \%$ of the total phenotypic variation for low-temperature germinability. Four additional QTL, accounted for $10.4-15.1 \%$ of the total phenotypic variation. The $O$. rufipogon alleles in all detected QTLs loci increased the low-temperature germination rate. No QTL associated with low temperature germinability has been detected near the qltg10 QTL in this study suggesting that qltg10 is a new QTL. The locus, qltg10 is of particular interest because of its independence from undesirable height and maturity effects. The DNA markers linked to the QTL for low temperature germinability would be useful in selecting lines with enhanced low temperature germinability in rice breeding program.
\end{abstract}

Key words : Rice, Low temperature germinability, QTL, Introgression lines

\section{Introduction}

Rice, being a temperature-sensitive crop, experiences yield loss due to low temperature (Mackill, 1995; Peyman and Hashem, 2010). About 15 million hectares of rice fields in 24 different countries is under threat from cold weather (Zeng et al., 2009). In Korea, rice experiences low temperature in northern and high altitude areas. Rice plants growing in these areas are regularly exposed to low temperature during different stages of growth i.e-vegetative, reproductive and ripening. The vegetative phase comprises of different stages namely the germination, seedling and tillering. Occurrence of low temperature stress either at germination or seedling stage affects seed germination and inhibits seedling

\footnotetext{
*Corresponding author: Tel: +82-42-821-5728

E-mail address: ahnsn@cnu.ac.kr
}

establishment leading to non-uniform crop maturation (Lou et al., 2007). In this regard, low temperature germinability is one of the major determinants for stable stand establishment in temperate, high altitude areas. In Korea, the direct seeding method has become increasingly important (Suh et al., 1999). Vigorous germination at low temperature is an important character to make stable establishment of seedlings in the direct seeding method.

In spite of the significance of low-temperature germinability in rice cultivation, the genetic mechanism of this trait is not well understood. Low-temperature germinability is considered to be a quantitative trait controlled by several genes as well as environmental factors. The linkage relationships between the genes for low-temperature germination and several morphological marker genes were examined using japonica linkage 
testers (Sasaki et al., 1973).

QTL studies were carried out to identify genes underlying low temperature stress at different developmental stages. These QTL analyses have revealed that lowtemperature germination is controlled by several genes (Suh et al., 1999; Miura et al., 2001; Fujino et al., 2004 ). Three QTLs for low-temperature germination were detected using $98 \mathrm{BC}_{1} \mathrm{~F}_{1}$ plants from a cross between Milyang23 and Hapcheonaengmi3 (Suh et al., 1999). Five QTLs for low-temperature germination were detected using 98 backcross inbred lines from a cross between Nipponbare and Kasalath (Miura et al., 2001). Three QTLs controlling low-temperature germinability were identified using 122 backcross inbred lines derived from a cross between temperate japonica varieties, 'Italica Livorno' and 'Hayamasari' and a major QTL on chromosome 3, qLTG3-1, explained $>30 \%$ of the total phenotypic variation in the mapping population and was localized to a 3.7-cM interval on chromosome 3 (Fujino et al., 2004). Subsequently, qLTG3-1 was cloned by a map-based strategy, and it was revealed that qLTG3-1 encodes a novel protein of 184 amino acids with unknown function (Fujino et al., 2008).

However, the number of reports on QTL analysis of the genes for the low temperature germinability in the Oryza rufipogon is quite rare. This study was conducted to detect QTLs for low temperature germinability using an introgression line population (ILs) derived from a cross between the Oryza rufipogon and the Korean japonica cultivar Hwaseongbyeo.

\section{Materials and methods}

\section{Plant materials}

$96 \mathrm{BC}_{4} \mathrm{~F}_{8}$ lines were employed in this study and the scheme of the population development is described in the paper by Yun et al. (2010). The wild rice O. rufipogon (IRGC 105491) was used as a pollen parent in crosses with O. sativa spp. japonica cv. Hwaseongbyeo, an elite Korean cultivar, followed by two successive backcrosses made with Hwaseongbyeo as the recurrent parent (RP). Among $820 \mathrm{BC}_{2} \mathrm{~F}_{1}$ plants, 172 individuals were selected based on phenotypic acceptability and selfed to generate $\mathrm{BC}_{2} \mathrm{~F}_{2}$ families for QTL analysis. For the present study, $98 \mathrm{BC}_{2} \mathrm{~F}_{2}$ lines, were selected as the basis for NILs development. These lines were backcrossed to Hwaseongbyeo two times and then allowed to self, generating $96 \mathrm{BC}_{4} \mathrm{~F}_{8}$ lines.

\section{Evaluation of low-temperature ger- minability}

The $96 \mathrm{BC}_{3} \mathrm{~F}_{8}$ lines and the 2 parents were evaluated for germinability at $15^{\circ} \mathrm{C}$. The geminability was determined using kernels that had been harvested 30 days after flowering, dried at $45^{\circ} \mathrm{C}$ for 2 days. Thirty seeds per line were placed on a filter paper in a $9 \mathrm{~cm}$ Petri dish, and $10 \mathrm{~mL}$ of distilled water was added. The dishes were placed in an incubator at $15^{\circ} \mathrm{C}$. The number of germinated seeds was counted daily in three replications. Data of the germination rate at 7 days after incubation were used for QTL analysis because a large parental difference was observed at 7 days. Also, the germinability of two parents was tested at $30^{\circ} \mathrm{C}$, the optimum temperature for germination in rice.

\section{DNA extraction and SSR analysis}

Fresh leaves were collected from the parents and $96 \mathrm{BC}_{3} \mathrm{~F}_{8}$ lines and used for DNA extraction. DNA was extracted from the fresh leaves by method as described in Causse et al., (1994). A total of 131 SSR markers polymorphic between Hwaseongbyeo and O. rufipogon were surveyed to detect the $O$. rufipogon introgressions in ILs,. The PCR reaction mixture containing 30ng of DNA, 1 unit of Taq polymerase, 2.5 uM each d NTP, $8 \mathrm{uM} \mathrm{F}, \mathrm{R}$ primer, and $10 \mathrm{x}$ PCR buffer (10 mM Tris - $\mathrm{HCl} \mathrm{pH} 8.3,50 \mathrm{mM} \mathrm{KCl}, 1.5 \mathrm{mM} \mathrm{MgCl} 2,0.1 \%$ Gelatin). Amplification was accomplished with the Thermo (BioRad) by using the step-cycle program set to denature at 
$94^{\circ} \mathrm{C}$ for 5 min, subsequent denaturing was at $94^{\circ} \mathrm{C}$ for $1 \mathrm{~min}$., annealing was $55^{\circ} \mathrm{C}$ for $1 \mathrm{~min}$., extension was $72^{\circ} \mathrm{C}$ for $1 \mathrm{~min}$., and step 2 through 4 were repeated for a total 35 cycles with a final extension at $72^{\circ} \mathrm{C}$ for 5 min. PCR products were run on $4 \%$ polyacrylamide denaturing gel for $2 \mathrm{hr}$ at 1800-2000V, and marker bands were revealed using the silver staining kit purchased from Bioneer Co., Korea (Panaud et al., 1996, www. bioneer.co.kr). Amplified DNA fragments showing clear polymorphism were used for the analysis of ILs and linkage mapping.

\section{QTL analysis}

The distance and orientation between markers were based on the previously published map (McCouch et al., 2002). A single point analysis (SPA) was performed to determine the effect of each marker on each trait. In SPA, QTL was declared if the phenotype was associated with a marker locus at $p<0.005$ or with two adjacent marker loci at $p<0.01$. Statistical analysis was done using the software Qgene (Nelson, 1997). The proportion of the total phenotypic variation explained by each QTL was calculated as an $\mathrm{R}^{2}$ value, from the regressions of each marker/phenotype combination

\section{Results}

\section{Comparison of the parents for ger- minability}

The gemination rate at $30^{\circ} \mathrm{C}$ for 3 days after incubation of Hwaseongbyeo and O. rufipogon was $100 \%$. No difference between the parents was observed at $30^{\circ} \mathrm{C}$. However, a difference between the parents was sown at $15^{\circ} \mathrm{C}$ condition (Fig. 1). O. rufipogon began germinating at the $3^{\text {rd }}$ day after the start of incubation, and 3 days thereafter almost all of the seeds germinated. In contrast, Hwaseongbyeo began germinating at the $6^{\text {th }}$ after the start of incubation, and 5 more days were needed for near-completion of all germination. The germination rate at $15^{\circ} \mathrm{C}$ for 7 days of 0 . rufipogon and Hwaseongbyeo was 93.3 and 21.7\%, respectively. The germination rates of ILs varied continuously from 0 to $48.3 \%$ at 7 days after incubation (Table 1, Fig. 2).

\section{Variation in the ILs}

The $96 \mathrm{BC}_{3} \mathrm{~F}_{8}$ lines and the 2 parents were evaluated for germinability at $15^{\circ} \mathrm{C}$. The germination rate at 7 days of $O$. rufipogon and Hwaseongbyeo was 93.3 and $21.7 \%$, respectively. ILs displayed a wide range of varia-

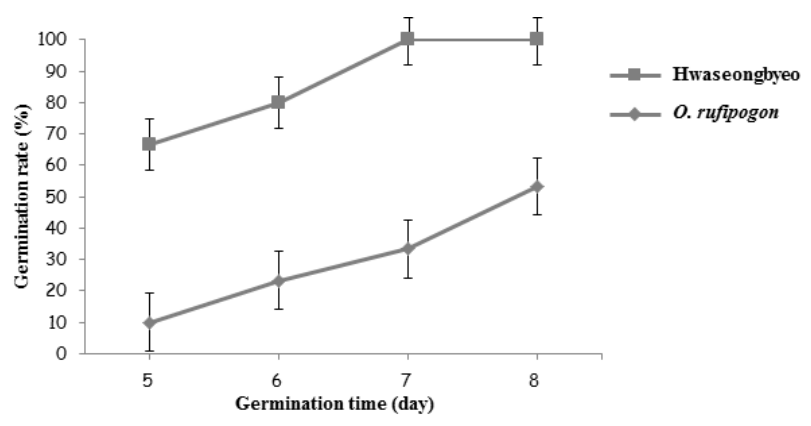

Fig. 1. Changes in the germination rate of the parents, $O$. rufipogon and Hwaseongbyeo at $15^{\circ} \mathrm{C}$.

* The circles and the boxes on each day show the means with standard deviation.

Table 1. Comparison of germinability at $15^{\circ} \mathrm{C}$ for the parents and ILs at 7 days after incubation

\begin{tabular}{ll}
\hline \multicolumn{1}{c}{ Lines } & \multicolumn{1}{c}{ Germinability (\%) } \\
\hline O. rufipogon & $93.3 \pm 9.4$ \\
Hwaseongbyeo & $21.7 \pm 11.8$ \\
$96 \mathrm{BC}_{3} \mathrm{~F}_{8}$ & Mean $13.3 \pm 12.75(0-48.3)^{1)}$ \\
\hline
\end{tabular}

1) Number in the parenthesis indicates range.

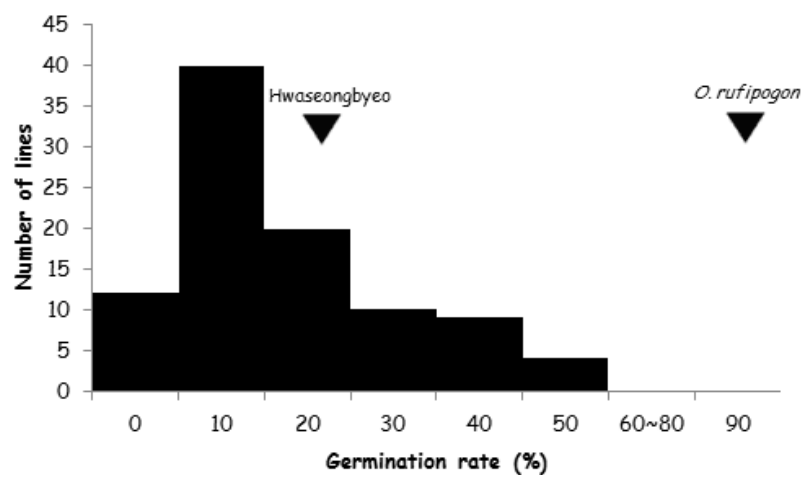

Fig. 2. Frequency distribution of germination rate at $15^{\circ} \mathrm{C}$ for ILs at 7 days after the start of incubation. 
tion from 0 to $48.3 \%$ at 7 days after incubation (Table 1, Fig. 2). The distribution of germinability in the ILs displayed continuous variation and transgressive lines that fell beyond the low mean of the parent were observed. Most of the lines showed similar level of germinability as the recurrent parent, Hwaseongbyeo.

\section{QTLs for low-temperature germinability}

For parental polymorphism screening with SSR markers,
296 of 460 (64.3\%) markers amplified scoreable and reproducible polymorphic bands. The genotypes of 96 ILs were surveyed by 131 markers. Five putative QTLS associated with low-temperature germinability were detected (Fig. 3, Table 2), and the O. rufipogon alleles in all detected QTLs loci increased the low-temperature germination rates. The QTL with a large effect, qltg10, was mapped at the marker RM147 and RM258 on the chromosome 10 (Fig. 3). The phenotypic variation explained by qltg10 was 19.2\%. Four additional QTLs,

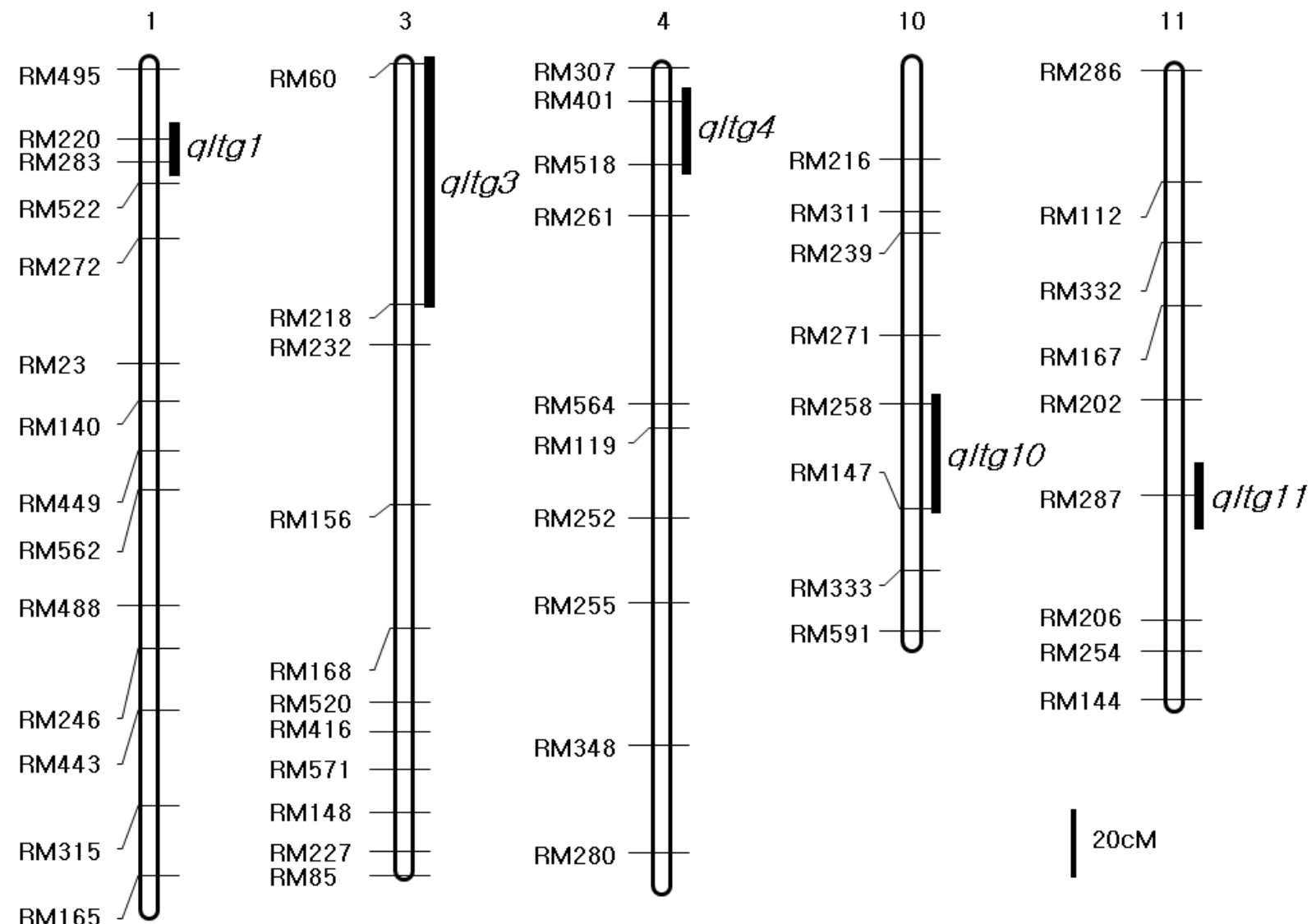

Fig. 3. Chromosomal locations of QTLs for low-temperature germinability in rice.

Table 2. Characteristics of QTLs for low-temperature germinability.

\begin{tabular}{cclllcc}
\hline QTL & Chr. & Marker interval & P & LOD & R2 & $\mathrm{AE}^{1)}$ \\
\hline qltg1 & 1 & RM220-RM283 & 0.01 & 2.35 & 12.1 & 5.1 \\
qltg3 & 3 & RM60-RM218 & 0.01 & 2.13 & 10.4 & 4.7 \\
qltg4 & 4 & RM401-RM518 & 0.0050 & 2.75 & 15.1 & 5.3 \\
qltg10 & 10 & RM258-RM147 & 0.00001 & 4.29 & 19.2 & 6.4 \\
qltg11 & 11 & RM287 & 0.0024 & 2.70 & 12.6 & 5.5 \\
\hline
\end{tabular}

${ }^{1)} \mathrm{AE}($ additional effect $)=($ O. rufipogon homozygote - Hwaseongbyeo homozygote $) / 2$ 
qltg1, qltg3, qltg4, and qltg11 on chromosomes 1, 3, 4, and 11 were detected and accounted for $12.1 \%$, $10.7 \%, 15.1 \%$, and $12.6 \%$ of the total phenotypic variation, respectively.

\section{Discussion}

Low-temperature germinability is one of the major determinants for stable stand establishment in the direct seeding method in temperate regions and at high altitudes of tropical regions. Previous genetic analyses have revealed that low-temperature germination is controlled by several genes (Sasaki et al., 1973; Miura et al., 2001). However, reports on genetic analysis of the genes for the low temperature germinability in wild species is rare.

In this study, a set of introgression lines carrying wild rice (O. rufipogon) segments were developed from a cross between Hwaseongbyeo and O. rufipogon. A total 131 SSR markers were used to detect chromosomal segments introgressed from O. rufipogon in 96 ILs. 5 QTLs related to low-temperature germinability were detected and the $O$. rufipogon alleles in all detected QTLs loci increased the low-temperature germination rates. Among the 5 QTLs, q1tg10 on chromosome 10 located near the SSR markers, RM258 and RM 147, explained a large part of the total phenotypic variation in the ILs population (19.2\%).

The location of the QTLs detected in this study was compared with that of the previous reports. A number of reports (Suh et al., 1999; Miura et al., 2001; Hou et al., 2004) detected QTL for low temperature germinability on chromosome 11. The QTL, qltg11 detected in this study shared the similar location as those identified in the 3 previous reports and this result suggests the possibility that these QTLs are allelic. Three QTLs for low-temperature germination have been identified by studies of crosses between temperate japonica Italica Livorno and Hayamasari (Fujino et al., 2004). One QTL, qLTG-3-1 near the marker GBR3001 was located on chromosome 3, and the Italica Livorno allele in this QTL increased low temperature germination (Fujino et al., 2004). Based on the chromosomal location, this QTL appears to correspond to the qltg3 detected in this study. Further analysis will be required to prove the allelic relationship between these QTLs. Also, the qltg4 QTL detected in this study appears to be the same as those identified in the japonica/indica population (Miura et al., 2001) and japonica/ japonica population (Fujino et al., 2004). However, the allelic relationship among these QTLs needs to be clarified. The other QTLs detected in this study appear to be different from those identified in the previous QTL studies.

So far, no QTL associated with low temperature germinability has been detected near the q1tg10 QTL in this study suggesting that qltg10 is a new QTL for low temperature germinability. Having identified a new QTL for increased low temperature germinability coming from an accession of $O$. rufipogon not widely utilized in rice breeding programs, the aim of our marker-assisted introgression program was to fix the favorable allele (from O. rufipogon) of this QTL in elite cultivars genetic background with as little additional O. rufipogon (donor) chromosome as possible. The locus, qltg10 is of particular interest because of its independence from undesirable height and maturity effects (data not shown). However, additional experiments are needed to determine the effect of q1tg10 on other agronomic traits.

\section{Acknowledgements}

This study was financially supported by research fund of Chungnam National University in 2011.

\section{References}

Causse MA, Fulton TM, Cho YG, Ahn SN, Chunwongse J, Wu K, Xiao J, Yu Z, Ronald PC, Harrington SE, Second G, McCouch SR, Tanksley SD. 1994. Saturated Molecular Map 
of the rice genome based on an interspecific backcross population. Genetics 138:1251-1274.

Fujino K, Sekiguchi H, Sato H, Kinuchi H, Nonone Y, Takenuchi Y, Ando T, Lin SY, Yano M. 2004. Mapping of quantitative trait loci controlling low-temperature germinability in rice (Oryza sativa L.). Theoretical and Applied Genetics 108:794-799.

Fujino K, Sekiguchi H, Matsuda Y, Sugimoto K, Ono K, Yano M. 2008. Molecular identification of a major quantitative trait locus, qLTG3-1, conferring low-temperature germinability in rice. Proceedings National Academy of Sciences, USA. 105:12623-12628.

Hou MY, Wang CM, Jiang L, Wan JM, Hidshi Y, Atsushi Y. 2004. Inheritance and QTL mapping of low temperature germinability in rice (Oryza sativa L.). Acta Genetics Sinica 31(7):701-706.

Lou Q, Chen L, Sun Z, Xing Y, Li J, Xu X, Mei H, Luo L. 2007. A major QTL associated with cold tolerance at seedling stage in rice (Oryza sativa L.). Euphytica 158:87-94.

Mackill DJ. 1995. Classifying japonica rice cultivars with RAPD markers. Crop Science 35: 889-894

McCouch SR, Teytelman L, Xu Y, Lobos KB, Clare K, Walton M, Fu B, Maghirang R, Li Z, Xing Y, Zhang Q, Kono I, Yano M, Fjellstrom R, DeClerck G, Schneider D, Cartinhour S, Ware D, Stein L. 2002. Development and mapping of 2,240 new SSR markers for rice (Oryza sativa L.). DNA
Research 9:199-207

Miura K, Lin SY, Yano M, Nagamine T (2001) Mapping quantitative trait loci controlling low-temperature germinability in rice (Oryza sativa L.). Breed Science 51:293-299.

Panaud O, Chen X, McCouch SR. 1996. Development of microsatellite markers and characterization of simple sequence length polymorphism (SSLP) in rice (Oryza sativa L.). Molecular General Genetics 252:597-607.

Peyman S, Hashem A. 2010. Evaluation of eighteen rice genotypes in cold tolerance at germination stage. World Applied Sciences Journal 11:1476-1480.

Sasaki T, Kinoshita T, Takahashi M. 1973. Estimation of the number of genes in the germination ability at low temperature in rice. Journal of Faculty Agriculture Hokkaido University 57:301-312.

Suh JP, Ahn SN, Moon HP, Suh HS. 1999. QTL analysis of low temperature germinability in a Korean weedy rice (Oryza sativa L.). Rice Genetics Newsletter 16:3-55.

Yun YT, Kim DM, Park IK, Chung CT, Seong YK, Ahn SN. 2010. Yun Development and Evaluation of QTL-NILs for Grain Weight from an Interspecific Cross in Rice. Korean Journal of Breeding Science 42(4):357-364.

Zeng YW, Yang SM, Cui H, Yang XJ, Xu LM, Du J, Pu XY, Li ZC, Cheng ZQ, Huang XQ. 2009. QTLs of cold tolerancerelated traits at the booting stage for NIL-RILs in rice revealed by SSR. Genes Genomics 31:143-154. 